\title{
Correction to: Diffusion, outcomes and implementation of minimally invasive liver surgery: a snapshot from the I Go MILS (Italian Group of Minimally Invasive Liver Surgery) Registry
}

\author{
Luca Aldrighetti ${ }^{1}$. Francesca Ratti ${ }^{1}$. Umberto Cillo ${ }^{2}$. Alessandro Ferrero ${ }^{3}$. Giuseppe Maria Ettorre ${ }^{4}$. \\ Alfredo Guglielmi ${ }^{5}$. Felice Giuliante ${ }^{6} \cdot$ Fulvio Calise $^{7}$. On behalf of the Italian Group of Minimally Invasive Liver \\ Surgery (I GO MILS)
}

Published online: 11 December 2017

(c) Italian Society of Surgery (SIC) 2017

\section{Correction to: Updates Surg (2017) 69:271-283 http://dx.doi.org/10.1007/ s13304-017-0489-x}

A technical error led to incorrect rendering of the author group in this article. The correct authorship is as follows:

Luca Aldrighetti, Francesca Ratti, Umberto Cillo, Alessandro Ferrero, Giuseppe Maria Ettorre, Alfredo Guglielmi, Felice Giuliante, Fulvio Calise on behalf of the Italian Group of Minimally Invasive Liver Surgery (I GO MILS)

The collaborators are: Raffaele Dalla Valle, AOU Parma, Parma; Vincenzo Mazzaferro, Istituto Nazionale Tumori, Milano; Elio Jovine, Ospedale Maggiore, Bologna; Luciano Gregorio De Carlis, Ospedale Niguarda Ca' Granda, Milano; Ugo Boggi, AOU Pisana, Pisa; Salvatore Gruttadauria, ISMETT, Palermo; Fabrizio Di Benedetto, AOU Policlinico di Modena, Modena; Paolo Reggiani, Ospedale Maggiore Policlinico, Milano; Stefano Berti, Ospedale Civile S.Andrea, La Spezia; Graziano Ceccarelli, Ospedale San Donato, Arezzo; Leonardo Vincenti, AOU Consorziale

The online version of the original article can be found under doi:http://dx.doi.org/10.1007/s13304-017-0489-x.

\footnotetext{
Luca Aldrighetti

aldrighetti.luca@hsr.it

1 IRCCS San Raffaele Hospital, Via Olgettina 60, 20132 Milan, Italy

2 Policlinico Universitario, Padua, Italy

3 Ospedale Mauriziano, Turin, Italy

4 Ospedale San Camillo Forlanini, Rome, Italy

5 Ospedale Universitario GB Rossi, Verona, Italy

6 Policlinico Gemelli, Rome, Italy

7 Ospedale Pinetagrande, Castel Volturno, Italy
}

Policlinico, Bari; Giulio Belli, Ospedale SM Loreto Nuovo, Napoli; Guido Torzilli, Istituto Clinico Humanitas, Rozzano; Fausto Zamboni, Ospedale Brotzu, Cagliari; Andrea Coratti, AOU Careggi, Firenze; Pietro Mezzatesta, Casa di Cura La Maddalena, Palermo; Roberto Santambrogio, AO San Paolo, Milano; Giuseppe Navarra, AOU Policlinico G. Martino, Messina; Antonio Giuliani, AO R.N. Cardarelli, Napoli; Antonio Daniele Pinna, Policlinico Sant'Orsola Malpighi, Bologna; Amilcare Parisi, AO Santa Maria di Terni, Terni; Michele Colledan, AO Papa Giovanni XXIII, Bergamo; Abdallah Slim, AO Desio e Vimercate, Vimercate; Adelmo Antonucci, Policlinico di Monza, Monza; Gian Luca Grazi, Istituto Nazionale Tumori Regina Elena, Roma; Antonio Frena, Ospedale Centrale, Bolzano; Giovanni Sgroi, AO Treviglio-Caravaggio, Treviglio; Alberto Brolese, Ospedale S.Chiara, Trento; Luca Morelli, AOU Pisana, Pisa; Antonio Floridi, AO Ospedale Maggiore, Crema; Alberto Patriti, Ospedale San Matteo degli Infermi, Spoleto; Luigi Veneroni, Ospedale Infermi AUSL Romagna, Rimini; Giorgio Ercolani, Ospedale Morgagni Pierantoni, Forlì; Luigi Boni, AOU Fondazione Macchi, Varese; Pietro Maida, Ospedale Villa Betania, Napoli; Guido Griseri, Ospedale San Paolo, Savona; Andrea Percivale, Ospedale Santa Corona, Pietraligure; Marco Filauro, AO Galliera, Genova; Silvio Guerriero, Ospedale San Martino, Belluno; Giuseppe Tisone, Policlinico Tor Vergata, Roma; Raffaele Romito, AOU Maggiore della Carità, Novara; Umberto Tedeschi, AOU Integrata Verona, Verona; Giuseppe Zimmitti, Fondazione Poliambulanza, Brescia. 